\title{
Aeroacoustic Noise Source Identification Using Irregularly Sampled LDV Measurements Coupled with Beamforming.
}

\author{
Miguel Garcia-Pedroche*, Gareth J. Bennett ${ }^{\dagger}$ \\ Trinity College Dublin, Ireland.
}

\begin{abstract}
This paper develops a technique which relates dynamic pressure measurements taken outside the hydrodynamic field of a low speed jet to single point, one component velocity measurements taken within the jet using a Laser Doppler Velocimetry (LDV) system. To improve the signal to noise ratio of the pressure measurement, a coherence based acoustic beamforming technique is applied and focussed on the LDV measurement volume in order to evaluate its contribution to the measured sound. As the pressure samples are acquired at regular time steps whereas the velocity at irregular time intervals dependent on the particle seeding, the calculation of the auto- and cross-spectra of the pressure and velocity measurements require special treatment. The signal processing procedures required to calculate these are described and implemented in the paper. To validate the proposed technique real experimental data was analysed. Laser Doppler Velocimetry measured velocity fluctuations created by rods placed in a jet. Synchronously, a planar random array focused on the same plane as the LDV measurement, measured pressure fluctuation outside the hydrodynamic field. The same set of data was then analysed using conventional and coherence based beamforming algorithms. The results from both techniques were compared. While conventional beamforming identified the contribution from the main sources within the flow, the coherence based method was able to single out the source related to the local measurement. The results shown demonstrated how coherence based beamforming, combined with LDV, can be a useful tool for aeroacoustic noise source investigations.
\end{abstract}

\section{Nomenclature}

$(\cdot)^{*} \quad$ Complex conjugate

$(\cdot)^{T} \quad$ Non-conjugate Transpose

$(\cdot)_{i} \quad$ for $i$-th receiver

$(\cdot)_{j} \quad$ for $j$-th receiver

\langle\rangle$\quad$ Time average

$\sigma^{2} \quad$ Variance

$\overrightarrow{\xi_{h}} \quad$ Vector containing the distance from all the receivers to a single focal point

$\vec{w} \quad$ Steering vector of length N

$B \quad$ Total number of time averages

$b \quad$ Single time data block

$f \quad$ Frequency $(\mathrm{Hz})$

$f_{m} \quad$ Mean data rate

$f_{r} \quad$ Re-sample rate

$G(f) \quad$ One sided spectrum

$H \quad$ Total number of focal points

$h \quad$ Single focal point

$j \quad$ imaginary unit

${ }^{*} \mathrm{PhD}$ Student, Dept. of Mechanical and Manufacturing Engineering, Trinity College, Dublin 2, Ireland.

${ }^{\dagger}$ Lecturer, Dept. of Mechanical and Manufacturing Engineering, Trinity College, Dublin 2, Ireland. AIAA Member. 
$k \quad$ Wave number

$L(f) \quad$ Low pass filter

$N \quad$ Total number of array receivers

$P_{c o h} \quad$ Coherence Beamformer output

$P_{c} \quad$ Conventional Beamformer output

\section{Introduction}

Whilst single far-field sound measurements quantify the sound field at a particular point in space, they are unable to shed specific information on the causal effects. Especially when studying complex sound fields, this type of measurement becomes hindered by poor signal-to-noise ratio and by the impossibility of identifying source locations. During the 1970's several techniques were developed aiming to extract specific information from complex sound fields. Many of these efforts were directed to the study of jet noise. Several researchers made use of acoustic mirrors. A microphone is located at the focal point close to a concave mirror while the second focal point is focused at the point of interest. The spatial filtering effect obtained allowed the location of individual sources within a complex sound field to be identified. As shown by Chu and Laufer ${ }^{1}$ and Grosche, ${ }^{2}$ significant results of the source distribution in a jet were obtained by the use of this technique. In a similar way, phased array microphone techniques were applied, among others by Billingsley. ${ }^{3}$ By means of manipulating the time delays between the focal point of interest and the receivers, phased arrays were able to mimic the effects of acoustics mirrors. As described by Sijtsma, ${ }^{4}$ at this time, due to limitations in data acquisition systems, array signal processing could not outperform acoustic mirrors in spatial resolution, frequency range and signal-to-noise ratio. Recently, thanks to advances in computer capacity and data acquisition systems the disadvantages of array processing over acoustic mirrors aforementioned are disappearing. These improvements, added to the much shorter times needed to obtain measurements when using arrays, have made array signal processing the preferred source location technique.

A different approach termed "causality correlation" was explored by Lee \& Ribner ${ }^{5}$ and Siddon. ${ }^{6}$ By means of cross-correlating a dynamic measurement within the flow (cause) and a far field sound pressure measurement (effect), Siddon proved that specific source information could be extracted from a complex flow. In most early experiments, the flow measurements were obtained by means of a hot-wire probe while a single microphone recorded pressure values in the far-field. In other cases, as shown by Hurdle ${ }^{7}$ the hot-wire was substituted by microphones placed directly on the flow. The main drawback with this type of technique was the intrusive nature of the flow measurement. As the probe altered the flow it was difficult to quantify the effects of such alteration. Siddon ${ }^{8}$ termed this uncertainty issue as the "Probe contamination factor". To avoid this issue, a number of non intrusive measuring techniques were applied. Laser Doppler Velocimetry (LDV) was used to measure velocity fluctuation within the flow, as shown by Schaffar ${ }^{9}$ and Richarz. ${ }^{10}$ Panda and Seasholtz ${ }^{11}$ made use of Rayleigh-scattering to measure flow density fluctuations. More recently Henning ${ }^{12}$ made use of Particle Image Velocimetry (PIV) as the in-flow measuring technique. All these techniques are based on the "causality correlation" approach described by Siddon. Siller et al. ${ }^{13}$ reports a variation to the "causality correlation" method. The single microphone in the far-field was substituted by a linear array of receivers. In Siller's case the near field or "reference" measurement was obtained by a dynamic pressure transducer (rumble probe) located in the combustion chamber of a full size aero-engine. A correlation was calculated between the reference measurement and the combined output of the linear array of receivers which was focused on the exit plane of the engine. The results identified a clear contribution from the combustion chamber to the far-field noise measured by the array under certain operating conditions. In the work of Henning et al., ${ }^{14}$ in-flow measurements obtained using PIV were correlated with the signals received by an array of receivers in the far-field. It should be noted that due to the low PIV sampling frequency, a phase averaging technique was applied to obtain the correlation measurements. The aforementioned two techniques, replaced the single receiver measurements in the far-field, as reported by Siddon, by that of an array of receivers. As shown by their results, the use of an array can improve the signal-to-noise ratio and with it the possibility of obtaining significant correlation values.

The work proposed in this paper describes a novel advancement to the "causality correlation" method. Here, the in-flow measurement (cause) will be obtained using LDV while simultaneously the acoustic field (effect) will be measured by an array of receivers. As the data rate obtained from an LDV probe is dependent on random seeding particles passing through the measuring volume, the signal must be resampled at equidistant steps prior to the application of standard signal processing techniques. In this paper sample-and-hold is 
used to achieve this. As documented by Adrian \& Yao ${ }^{15}$ and Boyer \& Searby ${ }^{16}$ this method introduces step noise and a low pass filter effect to the reconstructed signal. Due to this, a correction technique introduced by Simon \& Fitzpatrick ${ }^{17}$ and Fitzpatrick \& Simon $^{18}$ will be applied to estimate the auto and cross spectrum of the LDV reconstructed signal. The pressure data obtained by the array data will be processed using a coherence based beamforming technique. The aim of the present investigation is the development of the signal processing techniques necessary to obtain a frequency domain relationship between both measurements. The high mean data rate of the LDV signal will allow higher frequency and therefore shorter lengthscales to be investigated.

\section{Array signal processing}

Array signal processing, also known as "beamforming", is a well established noise source identification technique. As with acoustic mirrors, beamforming has been used to identify the location of noise sources in space. As described by Dudgeon, ${ }^{19}$ beamforming could be thought of as the spatial analogy to a band pass filter in the frequency domain. By selectively delaying and superimposing the receivers' signals, beamforming "steers" the maximum sensitivity of an array into a desired direction. Parting from this same principle, several algorithms have been developed in order to extract source information from array signals. Extensive literature covers this subject, but a particularly good example is documented by Sijtsma. ${ }^{4}$

\section{II.A. Array Design}

The first part of this project was the design of an array configuration suited for the purpose of the study. A number of variables had to be taken into account. As the area where the array is focused concentrates over the small volume measured by the LDV, a major concern was array resolution. Whilst increasing the array aperture improves resolution, large distances within receivers could restrict the array's working frequency range. This is found to be critical especially in the case of arrays with regularly spaced receivers. To maximize the working frequency range, while maintaining a large aperture, the response of different arrays with irregularly spaced receivers was simulated. The response of a randomized planar array was found to be the most favorable. The final microphone array design shown in figure 1(a) was chosen from a number of randomized array simulations. Figure 1(b) shows the response of this array using the conventional beamforming frequency domain algorithm to a simulated $3000 \mathrm{~Hz}$ monopole source located at $0.35[\mathrm{~m}]$ from the center of the array.

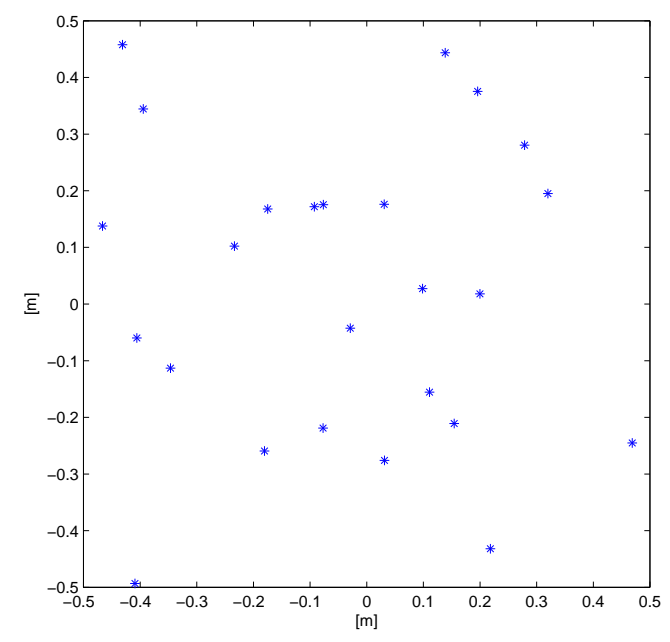

(a) Microphone layout

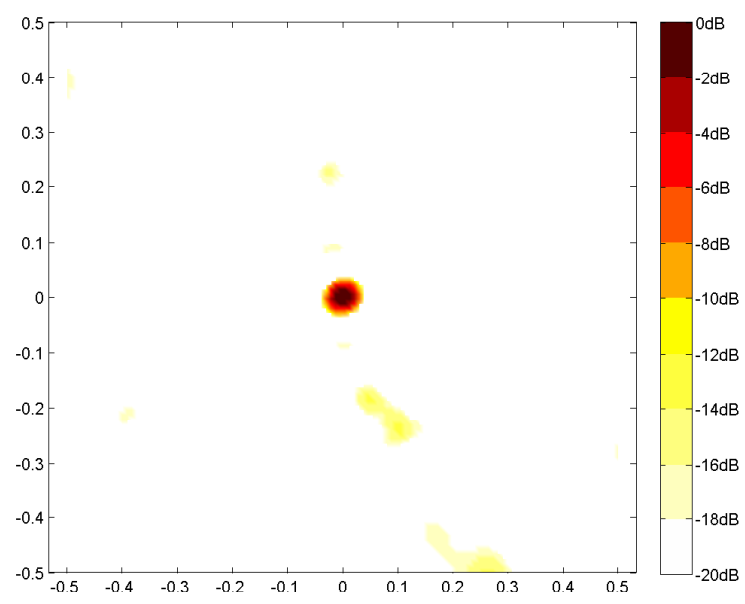

(b) Simulated array response for a $3000 \mathrm{~Hz}$ source at $0.35 \mathrm{~m}$

Figure 1. Microphone locations and array simulated response

The conventional beamforming algorithm used in this paper is as described by Johnson and Dudgeon. ${ }^{19}$ The pressure signals acquired by the array receivers are fourier transformed and a cross spectral matrix is 
formed as shown in equation (1).

$$
R_{(i, j)}(f)=\frac{1}{B} \sum_{b=1}^{B}\left[X_{i b}^{*}(f) X_{j b}(f)\right]
$$

where $X_{i b} \& X_{j b}$ are the complex pressures obtained by receivers $i$ and $j$ and $R_{i j}$ is the element of the cross spectral matrix in row $i$ and column $j$. The array focal point is selected by the steering vector shown in equation (2)

$$
\vec{w}=e^{-j k \overrightarrow{\xi_{h}}}
$$

Finally, the beamformer output for a single focal point is given by:

$$
P=\vec{w}^{*} R \vec{w}^{T}
$$

where $R$ is the Hermitian cross spectral matrix formed by all the $i t h$ and $j$ th elements in equation (1).

\section{II.B. Coherence beamforming}

Whilst conventional beamforming clearly identifies the location of sources within a narrow frequency band, a major drawback is that weaker sources within the same frequency range can be masked by louder ones or by side-lobes. To demonstrate this, two sources radiating incoherent white noise were located at $1[\mathrm{~m}]$ distance from the array as shown in figure 2. In all cases the SPL (Sound Pressure Level) generated by the source on the left was approximately $17 \mathrm{~dB}$ lower than the source on the right. The beamformer output shown is calculated for $4000 \mathrm{~Hz}$. As seen on figure 2(a) the conventional beamforming algorithm used, does not identify the weaker source on the left. The limiting effect that the side-lobes have on the beamformer dynamic range can also be observed. As is obvious, these factors can become an issue when trying to identify weaker sources in complex sound fields. In figure 2(b) the same set of data is now analysed using a coherence based beamforming technique. The coherence beamformer output shows now a clear contribution from the weaker source. Figure 2(b) clearly demonstrates that within the $4 \mathrm{~dB}$ dynamic range shown, the weaker source is now identified while the louder source on the right is suppressed.

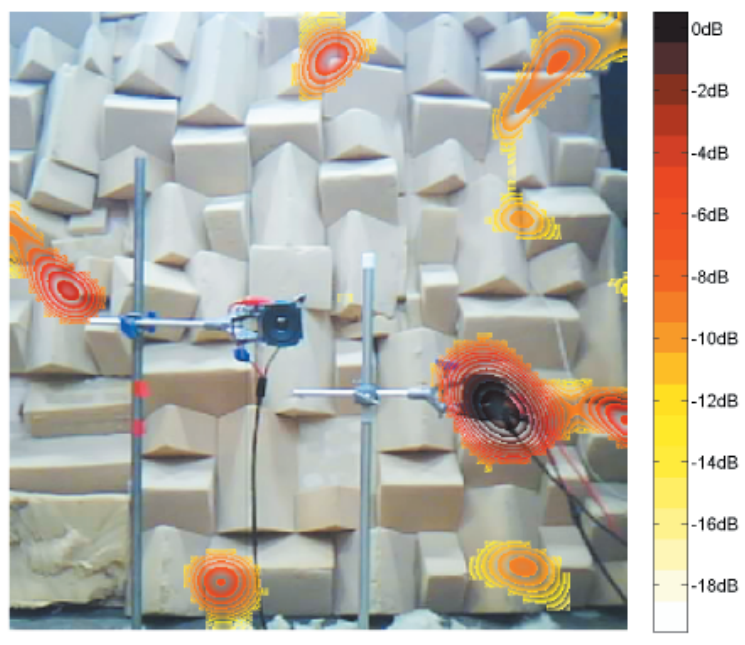

(a) Conventional Beamforming $(4000 \mathrm{~Hz})$

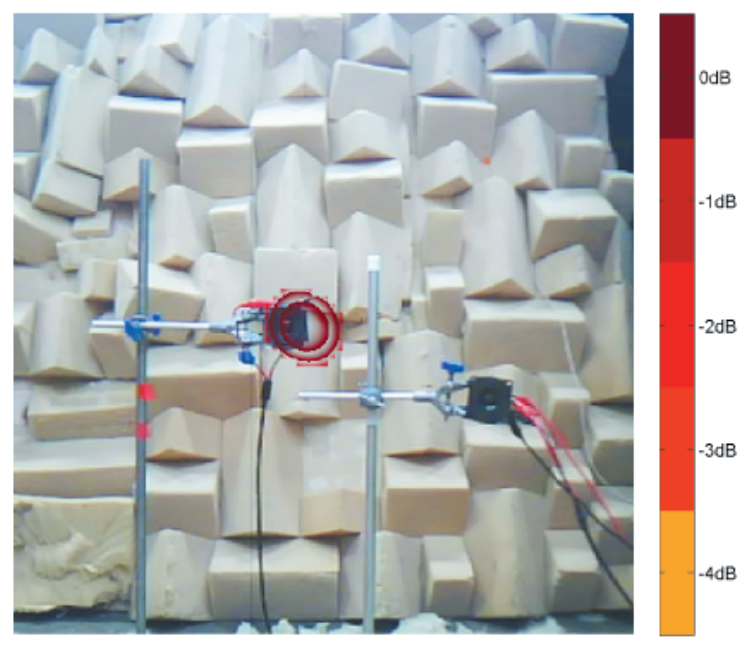

(b) Coherence Beamforming $(4000 \mathrm{~Hz})$

Figure 2. Conventional beamforming and Coherence based beamforming output

The coherence beamforming technique used here is as described by Guidati. ${ }^{20} \mathrm{~A}$ reference measurement of the source of interest is required, in this case the voltage signal driving the speaker shown on the left of figure 2 was used. This signal is then measured synchronously along with the array receivers. All signals are then Fourier-transformed and the complex values of all the pressure receivers are multiplied by the conjugate coefficients of the reference signal. The time averaged complex values obtained from this multiplication form the equivalent to the cross spectral matrix in conventional beamforming. The cross spectral matrix is now 
calculated as follows

$$
R c_{(i)}(f)=\frac{1}{B} \sum_{b=1}^{B}\left[X_{\text {Ref.signal }}^{*}(f) X_{j b}(f)\right]
$$

where $X_{\text {Ref.signal }} \& X_{j b}$ are the fourier transformed time signals of the reference measurement and the $j t h$ receiver and $R c_{(i)}$ is the $i_{t h}$ entry for the one dimensional cross spectral matrix. The coherence beamformer output for a single focal point is given by

$$
P_{c o h}=\vec{w}^{*} \cdot R c
$$

where $\vec{w}$ is the steering vector for focal point $h$ and $R c$ is the cross spectrum matrix formed in equation 4 . The coherence filtering algorithm is able to identify weak sources correlated to the reference measurement, even when these are buried within complex sound fields.

\section{II.C. Irregularly sampled data analysis}

Whilst LDV is a non-intrusive flow measuring technique, the data obtained is dependent on the rate of seeding particles crossing the laser beams. As a consequence, there is an inevitable irregularity to the samples acquired. Due to this, standard signal processing techniques can not be applied directly. To extract information from irregularly sampled signals a number of different techniques have been developed. Probably the most intuitive of these is the zero order interpolation or sample-and-hold. As shown in figure 3 , this technique reconstructs the data-set by holding the value of each data point until a new data point is validated. The reconstructed signal is then uniformly re-sampled so as to allow for conventional signal processing techniques to be applied.

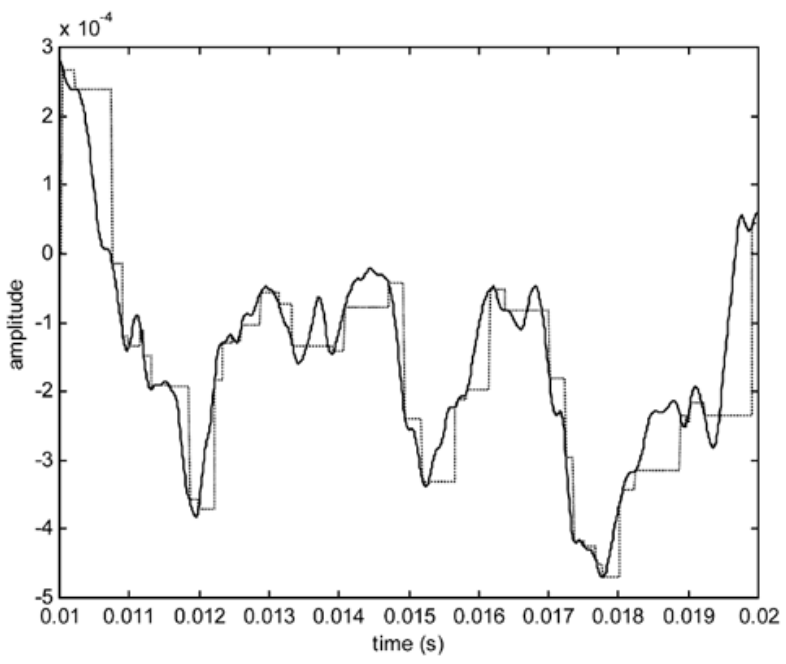

Figure 3. Sample and hold reconstruction

The errors associated with sample-and-hold have been documented by Adrian \& Yao ${ }^{15}$ and Boyer \& Searby. ${ }^{16}$ These are equivalent to the addition of step noise and a low pass filter effect. The system is illustrated in figure 4 . Here the step noise is represented by $s(t)$ and the low pass filter effect by $L(f), r(t)$ is the sample and hold reconstructed signal while $u(t)$ represents the true value of the velocity time series.

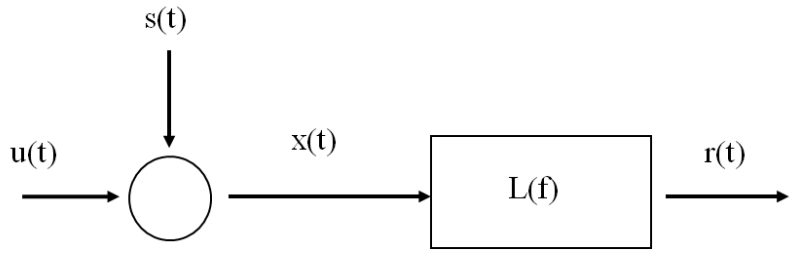

Figure 4. Schematic representation of Sample-and-hold reconstruction 


\section{II.D. Auto-spectrum estimation of an LDV signal}

As described by Simon \& Fitzpatrick ${ }^{17}$ and Fitzpatrick \& Simon $^{18}$ if the characteristics of the low pass filter are known and the step noise can be approximated, the spectrum $G_{r r}(f)$ can be corrected so as to obtain the estimate of the velocity signal auto-spectrum $G_{u u}(f)$. The correction for the low pass filter proposed by Fitzpatrick \& Simon ${ }^{18}$ is shown in equation (6). Here the discrete filter is a function of the mean data rate $f_{m}$ and the re-sample rate $f_{r}$.

$$
\left|L_{d}(f)\right|^{2}=\frac{f_{m}}{2 f_{r}} \cdot\left(\frac{1-e^{-2 f_{m} / f_{r}}}{1-2 \cos \left(2 \pi f\left(1 / f_{r}\right)\right)+e^{-f_{m} / f_{r}}+e^{-2 f_{m} / f_{r}}}\right)
$$

this is equivalent to the low pass recursive filter shown by Bendat and Piersol $^{21}$ as

$$
L_{d}(f)=\frac{1-e^{f_{m} / f_{r}}}{1-e^{-f_{m} / f_{r}} e^{-j 2 \pi f_{m} / f_{r}}}
$$

As the spectrum of the step noise introduced by sample-and-hold is white, this can be statistically estimated by calculating the variances of the signals. Even though is not possible to determine the variance of $u(t)$, Simon \& Fitzpatrick ${ }^{17}$ have shown that this is equivalent to the variance of the reconstructed signal $\sigma_{r}^{2}$. Therefore the variance of the step noise can now be found from:

$$
\sigma_{s}^{2}=\sigma_{x}^{2}-\sigma_{u}^{2}
$$

The step noise correction constant over $\mathrm{N}$ points is now given by:

$$
G_{s s}=\frac{1}{N}\left(\sum \frac{G_{r r}(f)}{|L(f)|^{2}}-\sum u(t)^{2}\right)
$$

An estimate of the true spectrum $G_{e e}$ can now be found by subtracting the step noise spectrum from the filter corrected spectrum.

$$
G_{e e}(f)=\frac{G_{r r}(f)}{|L(f)|^{2}}-G_{s s}
$$

An example of the use of this technique on simulated data is presented by Simon \& Fitzpatrick. ${ }^{17}$ In order to simulate LDV data an original signal was sampled using two irregular mean data rates $\left(f_{m}\right)$ of $500 \mathrm{~Hz}$ $\& 5 \mathrm{kHz}$. The set of irregular data points was reconstructed by means of sample-and-hold interpolation and re-sampled at a frequency of 50kHz. Figure 5(a) presents the autospectrum of the original set of data $G_{u u}$ against the autospectrum $G_{r r}$ directly estimated from the sample-and-hold using a $f_{m}$ of $500 \mathrm{~Hz}$. Figure 5 (a) clearly shows the low pass filter effect and the high frequency bias introduced by the sample-and-hold. Figure 5(b) shows the effects of applying a higher $f_{m}$ value $(5000 \mathrm{~Hz})$ and the correction proposed in equation 10. It is shown that the original signal can be correctly estimated from the irregularly sampled signal up to about a frequency corresponding to the mean data rate $f_{m}$. 


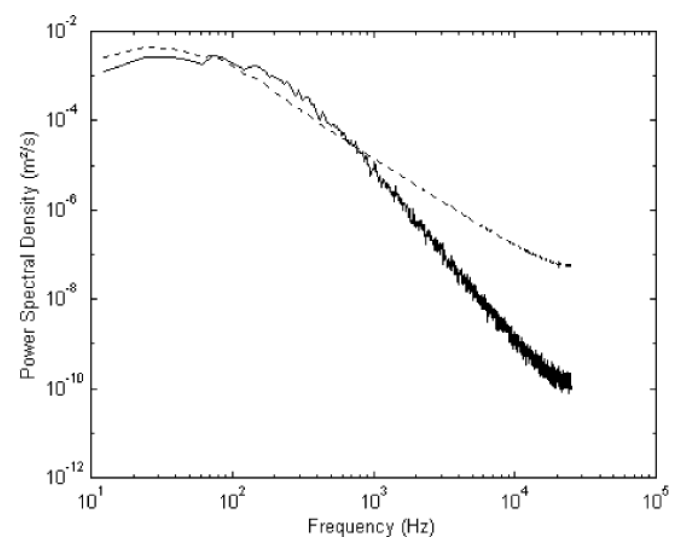

(a) $\operatorname{Guu}_{(\text {solid })} \& \operatorname{Grr}_{(\text {dots })}\left(f_{m}=500 H z\right)$

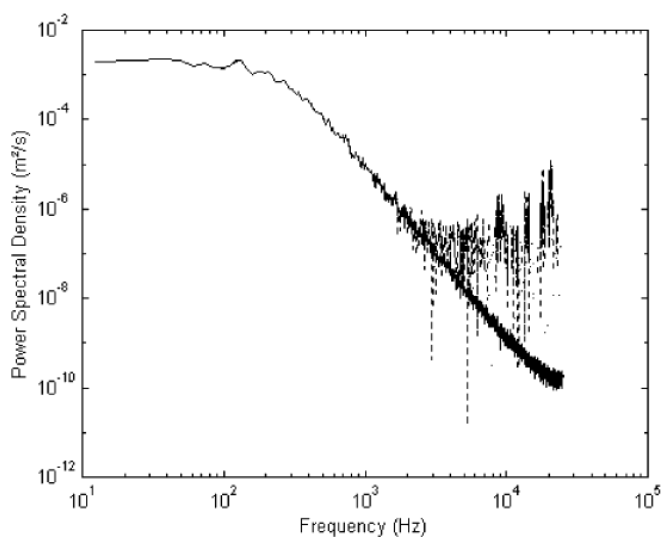

(b) $\operatorname{Guu}_{(\text {solid })} \& \operatorname{Grr}_{(\text {dots })}\left(f_{m}=5000 H z\right)$

Figure 5. PSD of the original signal and the direct sample-and-hold reconstruction for mean sample rates of 500Hz \& $5000 \mathrm{~Hz}$

\section{II.E. Cross-spectrum estimation between and LDV and a regularly sampled signal}

For all the tests carried out in this paper, the cross spectrum between an LDV measurement and a regularly sampled microphone signal was required. The schematic representation of this particular case is presented in figure 6 ,

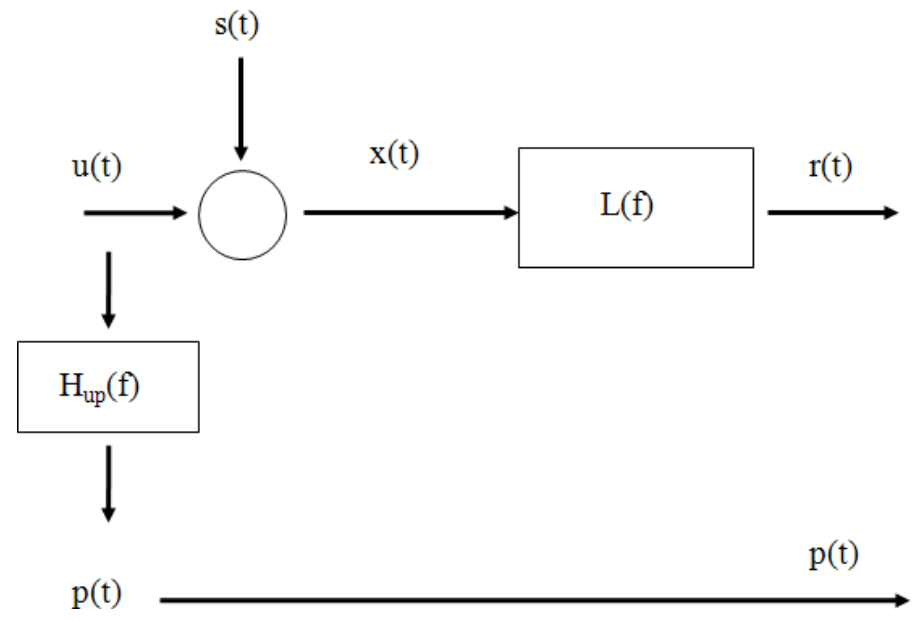

Figure 6. Schematic representation of sample-and-hold for LDV and a regularly sampled microphone signal

where $\mathrm{p}(\mathrm{t})$ represents a single regularly sampled microphone signal and $H_{u p}$ represents the frequency response function between the two measurements.

As shown by Fitzpatrick and Simon ${ }^{18}$ the relation between the elements in figure 6 can be written in the frequency domain as:

$$
\begin{aligned}
P(f) & =H_{u p}(f) U(f) \\
R(f) & =L(f)\{U(f)+S(f)\}
\end{aligned}
$$

The cross spectrum between the LDV and the microphone signal becomes:

$$
\begin{aligned}
G_{r p}(f) & =\left\langle R^{*}(f) P(f)\right\rangle \\
& =L^{*}(f)\left\{\left\langle U^{*}(f) P(f)\right\rangle+\left\langle S^{*}(f) P(f)\right\rangle\right\}
\end{aligned}
$$

As the noise due to sample and hold $s(t)$ is uncorrelated with the microphone signal $p(t)$ the term $\left\langle S^{*}(f) P(f)\right\rangle$ in equation (12) reduces to zero, giving: 


$$
G_{r p}(f)=L^{*}(f) G_{u p}(f)
$$

If the estimate of $L(f)$ is given by equation (7) the estimate of the cross spectrum between the true LDV and the pressure signal, $G_{\text {eup }}(f)$, can be found as

$$
G_{\text {eup }}(f)=\frac{G_{r p}(f)}{L^{*}(f)}=G_{x p}(f)
$$

\section{II.F. Coherence Beamforming using an LDV signal as reference value}

As shown in section II.B having a reference signal of the area of interest enables coherence beamforming to separate and/or identify sound sources where a conventional beamforming algorithm alone would fail. In order to use an LDV signal as the reference measurement of the area of interest, there is the need to precondition the LDV output prior to the application of the beamforming algorithm. For this, the parameters conditioning the frequency resolution of the fft's of the signals must be set. These are chosen so as to obtain equal frequency resolution in both, pressure and velocity signals. As an example, for all tests shown here the sampling frequency used for the pressure data acquisition was $12 \mathrm{kHz}$, while the mean sample rates obtained with the LDV varied from, approximately, $20 \mathrm{kHz}$ to $40 \mathrm{kHz}$. The sample-and-hold resample frequency applied for the LDV data was fixed at $120 \mathrm{kHz}$. Equal frequency resolution was set by choosing time windows of length 512 and 5120 samples for the pressure and LDV signals respectively. The data was then fourier transformed and the LDV signal was low pass filtered using expression (7). This preconditioning process is shown in figure 7

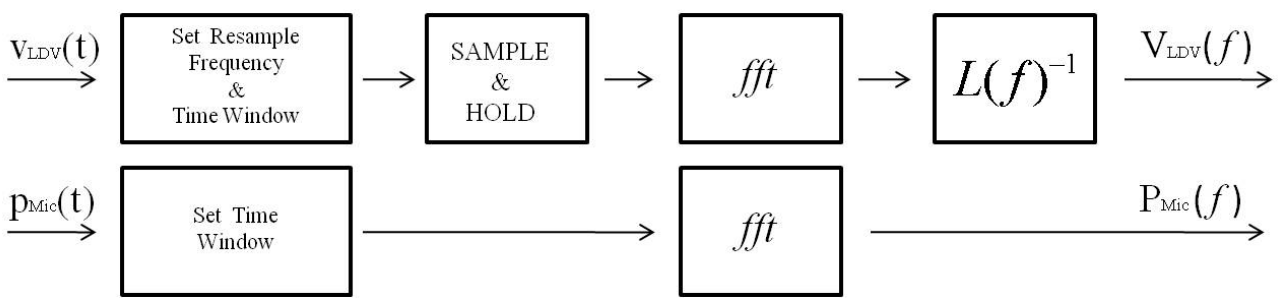

Figure 7. Signal conditioning schematic representation

The cross spectrum between both, pressure and velocity signals can now be calculated as follows

$$
G_{\left(V_{L D V} P_{M i c}\right)}(f)=2\left\langle\left(V_{L D V}(f)\right)^{*} \cdot\left(P_{M i c}(f)\right)\right\rangle
$$

Once equation (15) is computed between the LDV and all the microphone signals, this forms the cross spectral matrix used for beamforming. The beamformer output for a single focal point $h$ is now given by:

$$
P_{c o h}=\vec{w}^{*}\left(\begin{array}{c}
2\left\langle\left(V_{L D V}(f)\right)^{*} \cdot\left(P_{M i c_{1}}(f)\right)\right\rangle \\
2\left\langle\left(V_{L D V}(f)\right)^{*} \cdot\left(P_{M i c_{2}}(f)\right)\right\rangle \\
\vdots \\
2\left\langle\left(V_{L D V}(f)\right)^{*} \cdot\left(P_{M i c_{N}}(f)\right)\right\rangle
\end{array}\right)
$$

where $w$ is the steering vector $e^{-j k \overrightarrow{\xi_{h}}}$

\section{Experimental Set up}

The experiments were performed in the subsonic open jet facility at Trinity College Dublin as described by Kerherve et al. ${ }^{22}$ A $50 \mathrm{~mm}$ diameter jet discharges into quiescent air from a plenum through an elliptical 
bell-mouth with jet exit velocities from $30 \mathrm{~m} / \mathrm{s}$ to $80 \mathrm{~m} / \mathrm{s}$ and associated Reynolds numbers based on the exit nozzle diameter between $1 \times 10^{5}$ and $3 \times 10^{5}$. An arrangement of honeycombs and screens followed by the bell-mouth ensures that the flow in the nozzle has a low turbulence level and is aerodynamically uncoupled with the upstream fan. A $470 \mathrm{~mm}$ length nozzle configuration was used for the current tests. A planar 25 microphone random array with a webcam at its center was located below the jet as shown in figure 8. For all experiments the primary flow jet velocity was fixed at approximately $80 \mathrm{~m} / \mathrm{s}$.

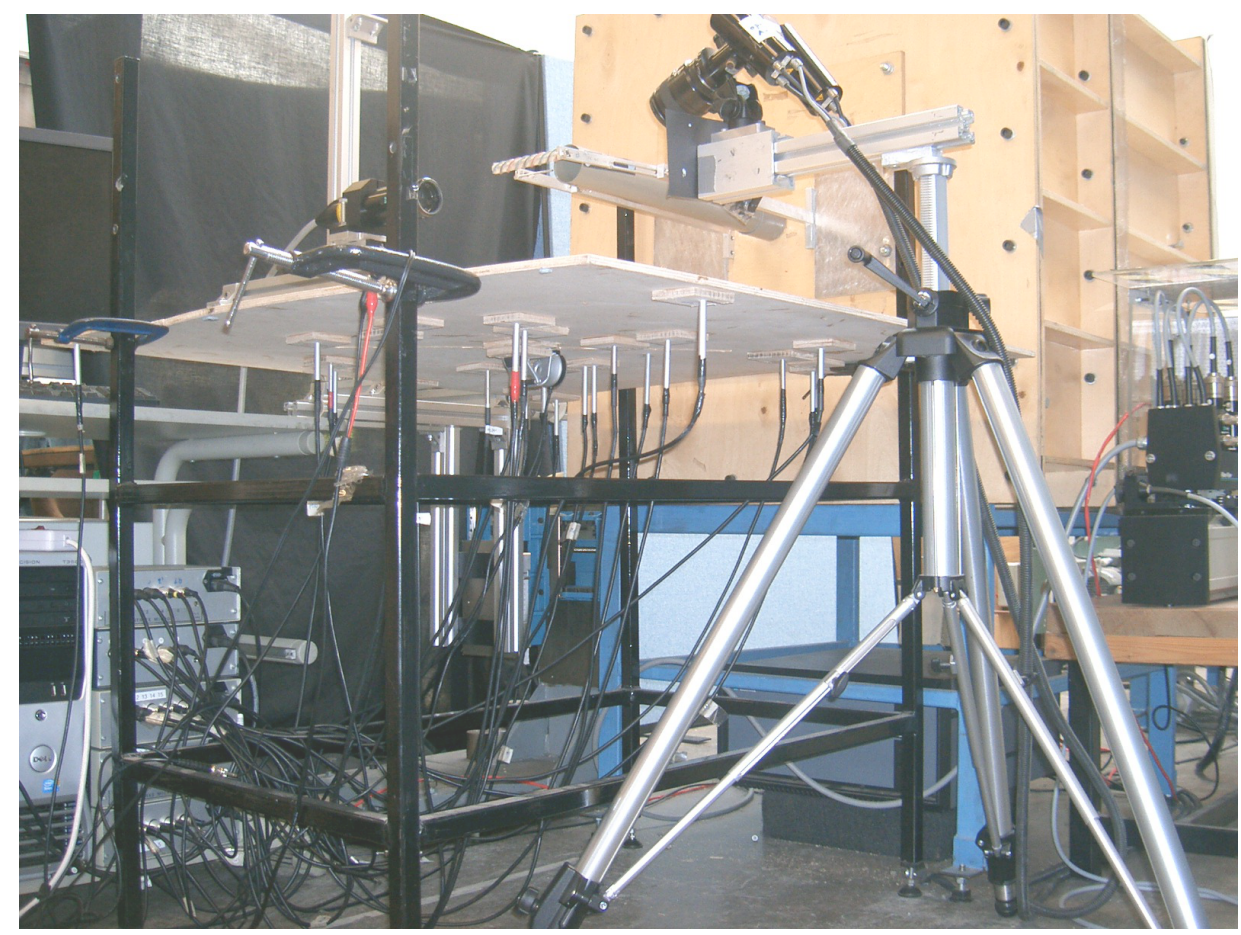

Figure 8. Planar array located below the measured volume

The chosen 25 channel planar random array configuration is described in section II.A. The array data was acquired using a National Instruments 36 channel, 24 bit data acquisition system. Each channel is simultaneously sampled and automatically low pass filtered to avoid aliasing. The acquired data was stored on the PC, ready for post processing. Two pairs of $4 \mathrm{~mm}$ threaded rods were located in the wake of the jet stream as shown in figures 9 and 10. The rod diameters and separation within each pair of rods was kept equal. The aim was to produce two known noise sources of approximately the same level and with overlapping frequencies. The array location and the rod pair orientation was chosen to avoid focusing the array on a dipole source. 


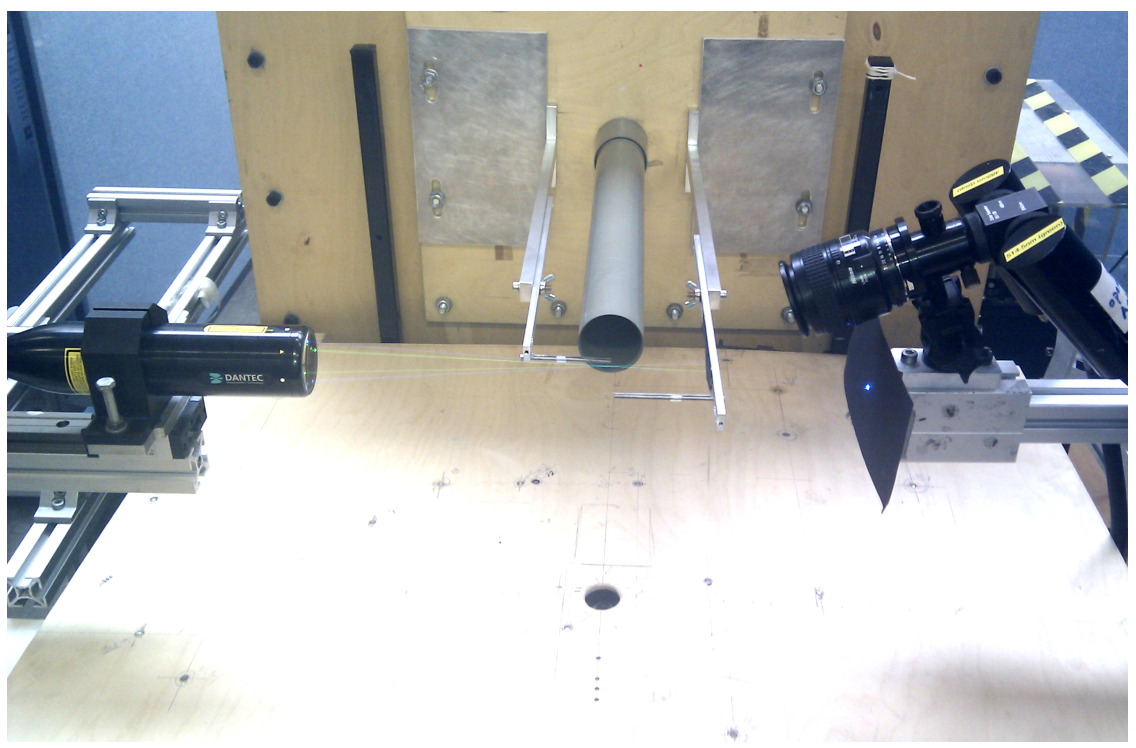

Figure 9. Planar array located below the measured volume

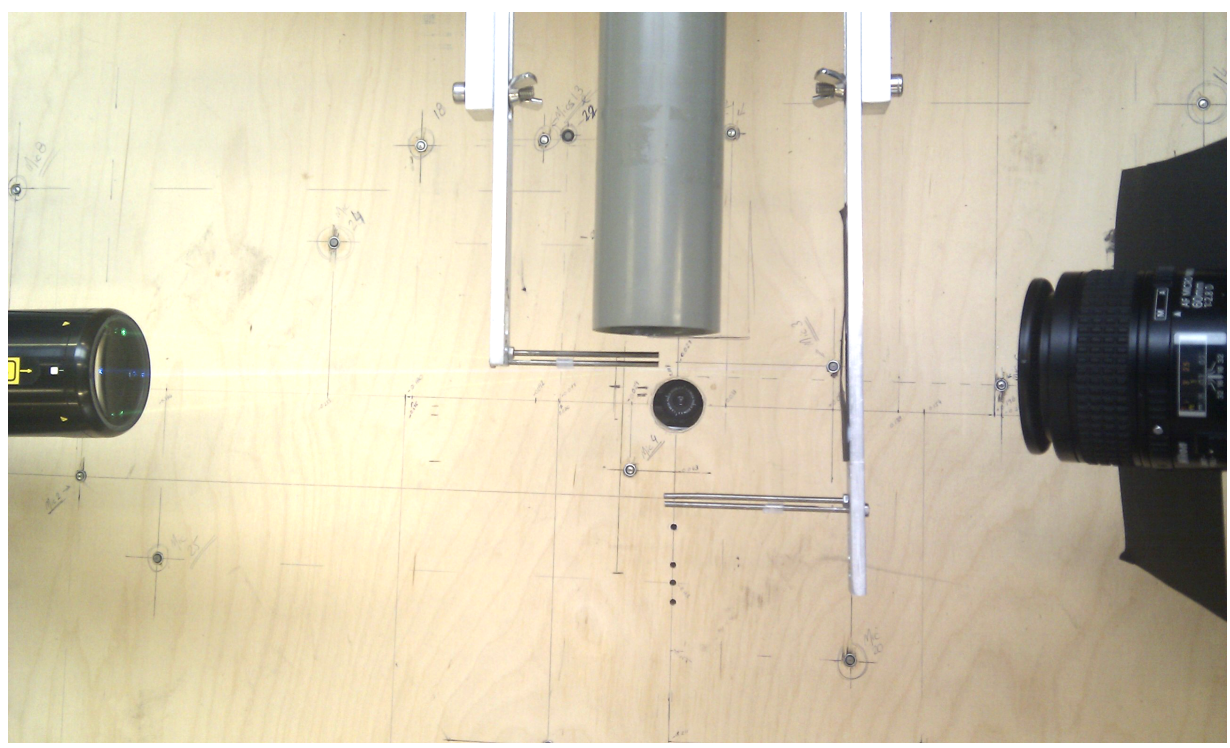

Figure 10. Top view of the test set up

The LDV system consists of a $500 \mathrm{~mW}$ Argon-ion laser and a dual beam Dantec optical head with blue $(488 \mathrm{~mm})$ and green $(514 \mathrm{~nm})$ wavelengths for vertical $(\mathrm{w})$ and longitudinal $(\mathrm{u})$ velocity components respectively. Dimensions of the measurement volumes for both wavelengths are $(0.12 \times 0.12 \times 1.6) \mathrm{mm}^{3}$, the largest dimension corresponding to the measurement volume length. To optimise data sample rates and signal-to-noise ratios, the system was operated in the forward scatter mode. As shown in figure 9 the receiving lens was located in front of the beam head at an off-axis angle. A pinhole of approximately $0.1 \mathrm{~mm}$ diameter in the receiving optic is used to collect the scattered light. The LDV and audio data acquisition systems were synchronized by an external trigger. The LDV data collected was analysed using a Dantec burst spectrum analyser type BSA F50 and stored on the PC ready for post processing. The tests carried out comprised of two main parts, for which the velocity of the jet was kept constant at approximately 80 $\mathrm{m} / \mathrm{s}$. The first section of the tests focused the LDV volume on the vortex wake created by the set of rods in close proximity to the nozzle exit as shown in figure 11(a). Simultaneously, the microphone array located below the test piece, at a distance of $0.25 \mathrm{~m}$, recorded the sound field. The second part of the test, was a repetition of the first set of tests, the only variation being that the LDV volume was located on the wake created by set of rods further away from the nozzle exit as shown in figure 11(b). Even though it can not be 
appreciated in figure 11, in order to minimize possible flow-acoustic interaction, the sets of rods were offset by approximately $1 \mathrm{~cm}$ and did not overlap in the horizontal plane.

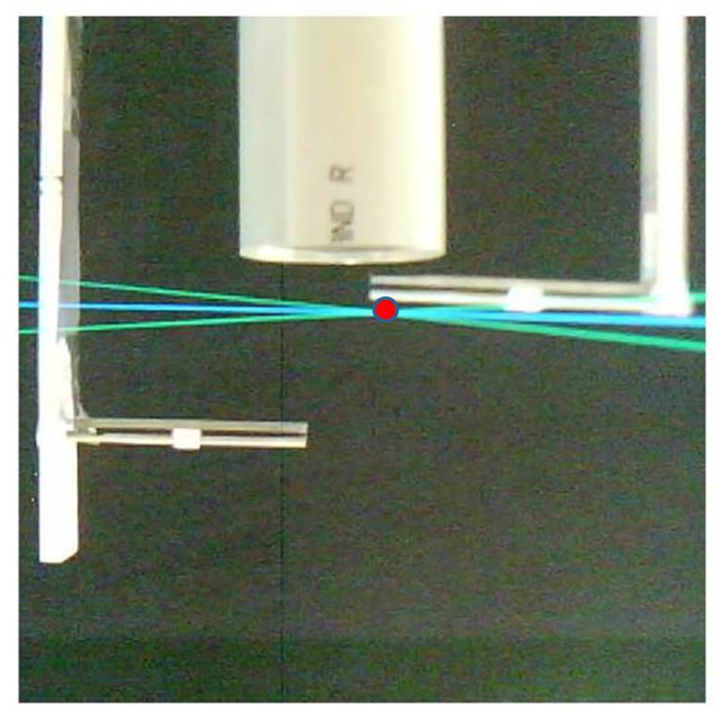

(a) Upstream

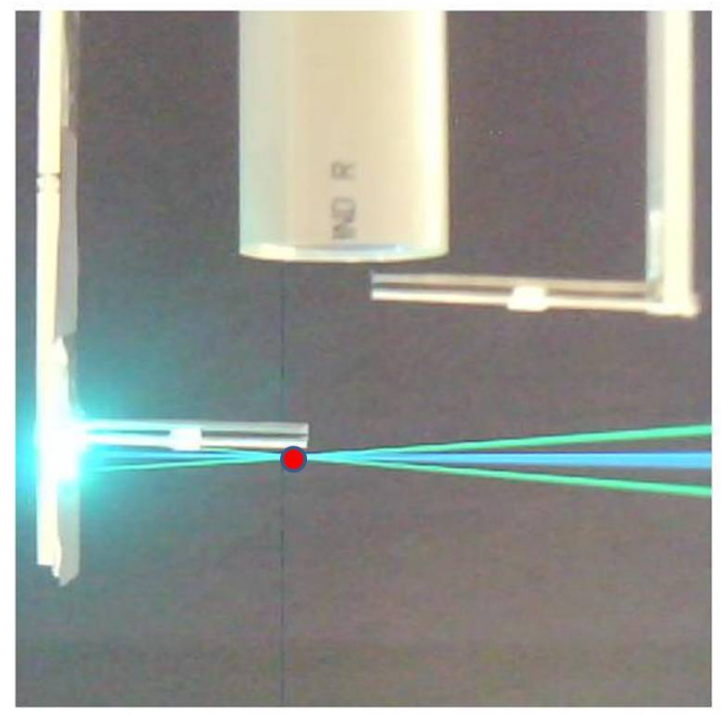

(b) Downstream

Figure 11. Test set-ups. The red dot indicates the location of the LDV measurement volume for each test

\section{Experimental Results}

The tonal characteristics of the sound generated by the set of rods within the jet's flow are shown in figure 12. Figure 12(a) shows the background noise characteristics of the jet in free flow conditions (no rods) whilst figure 12(b) presents the same result when the sets of rods depicted in figure 11 are in the flow. Whilst there are clear similarities in the region below $2000 \mathrm{~Hz}$, the narrow band energy centered at around $3844 \mathrm{~Hz}$ can be attributed to the addition of the sets of rods within the flow. Based on these results, the beamforming frequency for all measurements hereafter was fixed at $3844 \mathrm{~Hz}$.

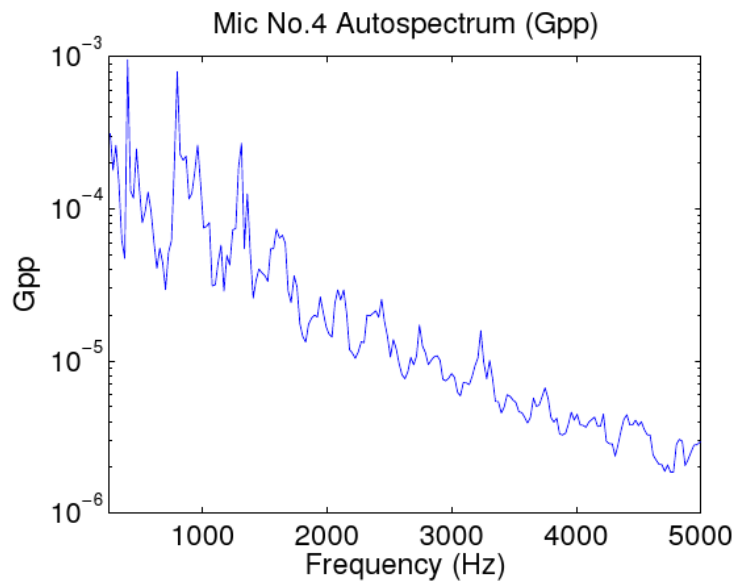

(a) No rods in the flow

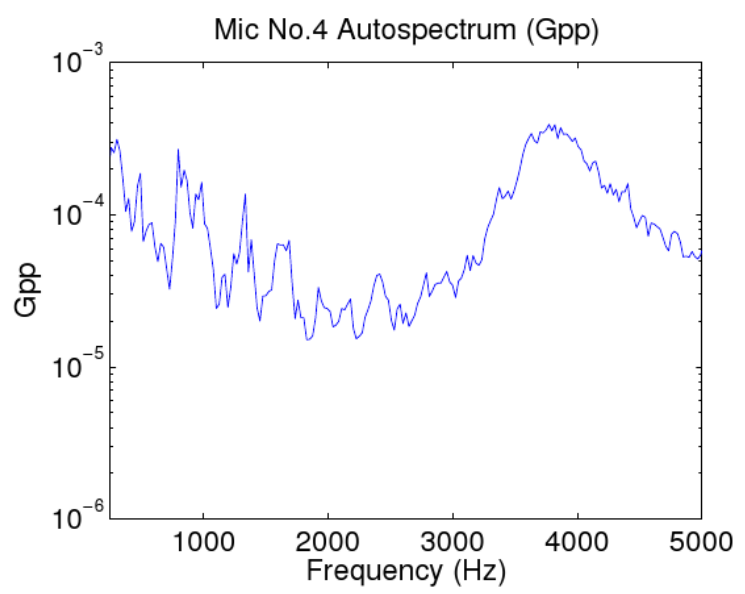

(b) With rods in place

Figure 12. Single microphone autospectrum with and without the set of rods

\section{IV.A. LDV Volume at the upstream location}

The results obtained for the set-up depicted in figure 11(a) are shown next. Figure 13 presents the output of the conventional beamforming algorithm. This technique, clearly identifies two separate main sources 
corresponding to the location of each pair of rods.

In order to apply the coherence beamforming algorithm, the reference signal was attained by the LDV volume located in the near wake of the upstream rods as shown by the red dot in figure 11(a). Figure 14 presents the step noise and filter corrected autospectrums of the flow velocities (u) \& (w) at this location. In both plots, but more clearly in the case of the $(\mathrm{w})$ component, there is a broad peak of energy at around $3800 \mathrm{~Hz}$. Whilst $(\mathrm{u})$ could be expected to be the principal component of velocity at this location, the magnitude of the $(\mathrm{w})$ component of flow velocity is greater. The higher value of the $(\mathrm{w})$ component indicates that the vorticity created by the set of rods, generated greater vertical flow motion at the location of the LDV measurement. The same broad peak of energy can be seen in the magnitude of the cross spectrum Gup \& Gwp in figures 15(a) \& 16(a). This result, clearly shows the relation between the flow disturbance measured by the LDV (cause) and the acoustic pressure (effect) at the array location. Figures 15(b) \& 16(b) present the result of the coherence beamforming algorithm when both of the local measurements $(\mathrm{u}) \&(\mathrm{w})$ are used as the reference signal in equation (16). In both cases, this technique is able to single out the source upstream. In other words, coherence beamforming shows the spatial location(s) of the causal effects if these are directly correlated to the reference signal. This could explain why, even though the LDV measurement was obtained behind the set of rods, the main source location was still identified towards the center of the set of rods.

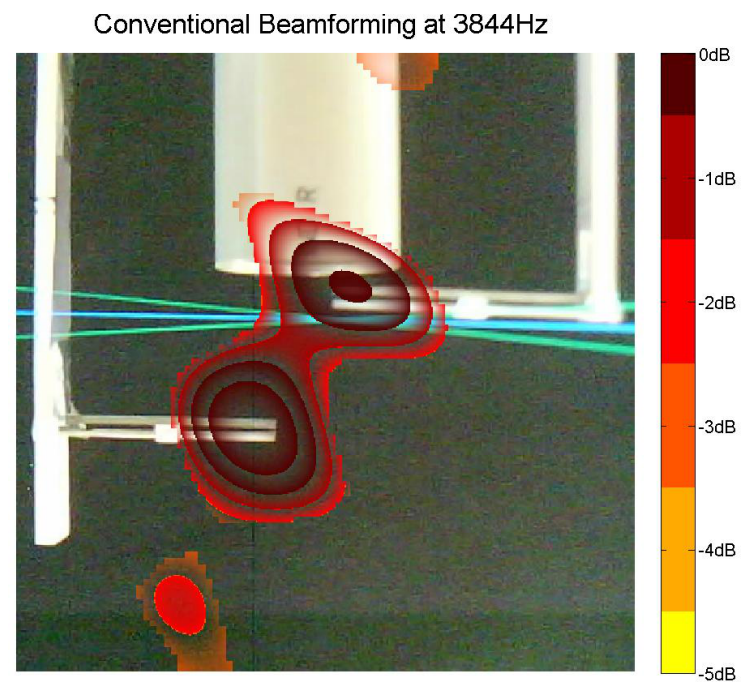

Figure 13. Conventional Beamforming

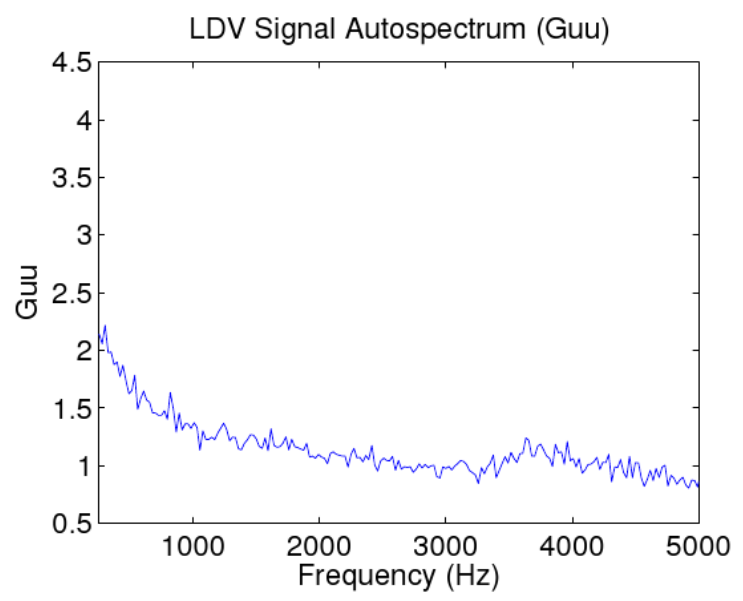

(a) Velocity autospectrum Guu

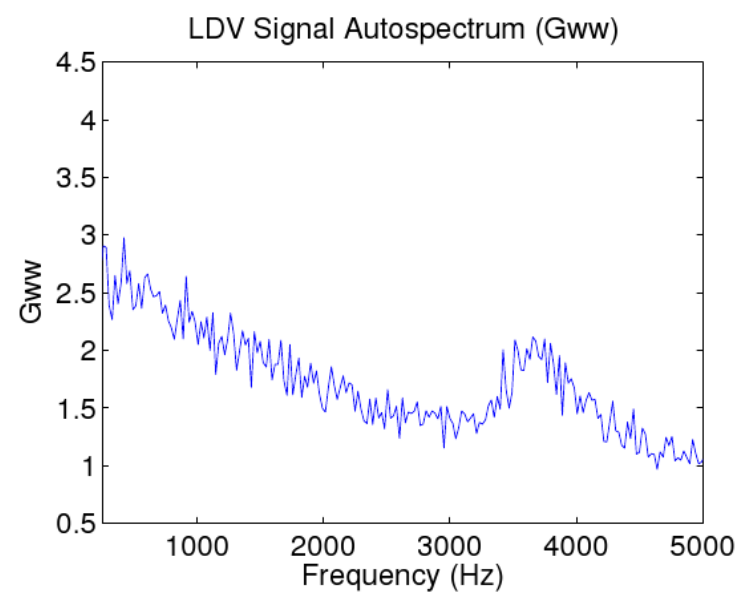

(b) Velocity autospectrum Gww

Figure 14. 


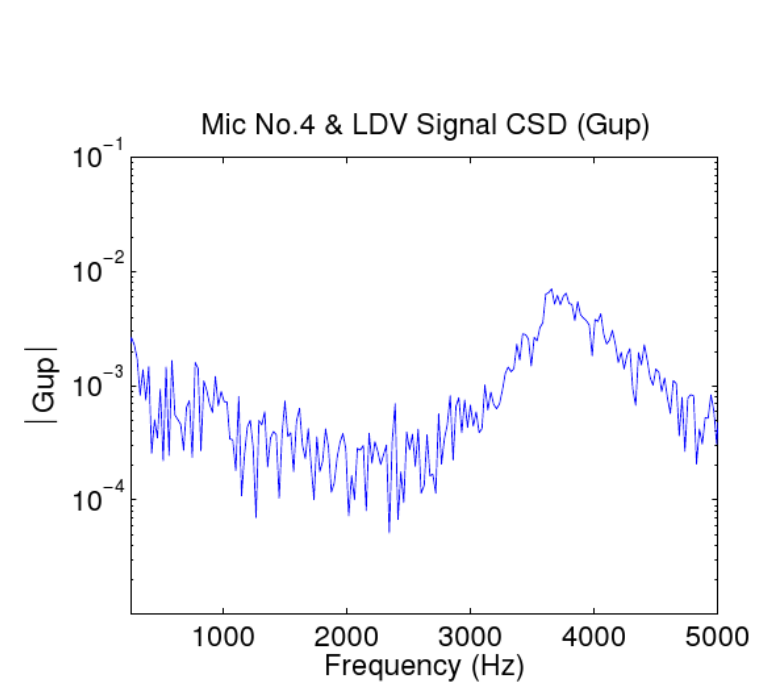

(a) Cross spectrum between the LDV (u) signal and Mic No.4

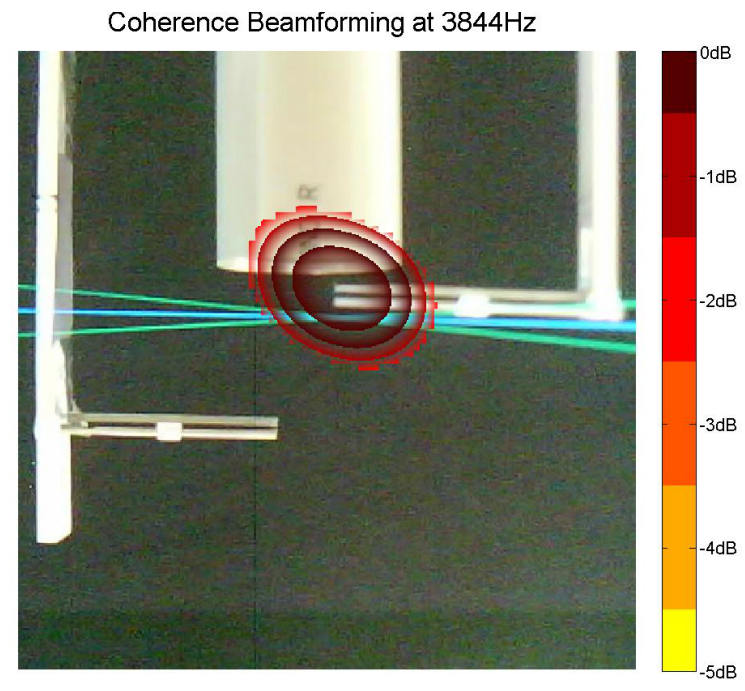

(b) Coherence Beamforming LDV (u) component as reference

Figure 15.

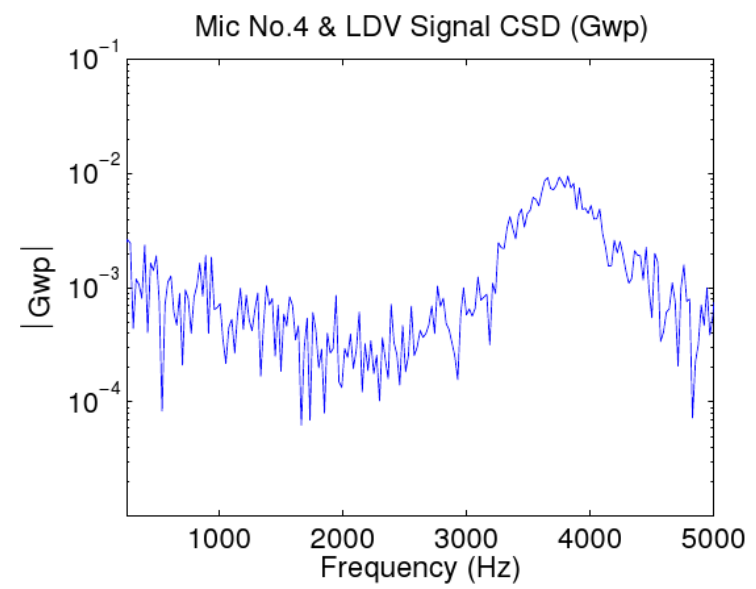

(a) Cross spectrum between the LDV (w) signal and Mic No.4

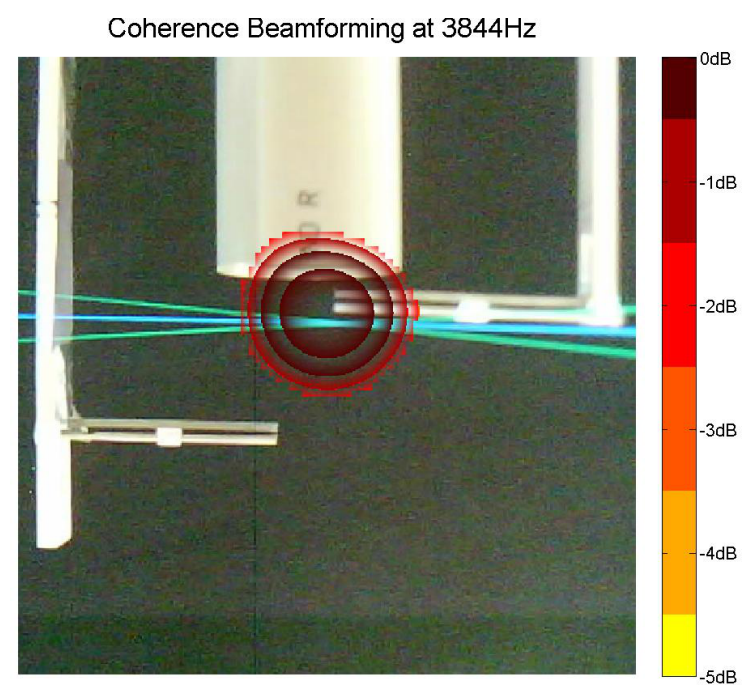

(b) Coherence Beamforming LDV (w) component as reference

Figure 16.

\section{IV.B. LDV Volume at the downstream location}

In the second part of the test, the LDV measurement volume was located behind the set of rods downstream as shown in figure 11(b). As in the previous case, the conventional beamforming output shown in figure 17, identifies the upstream and downstream sources simultaneously whilst coherence beamforming, figures 19(b) \& 20 (b), as in the previous case, is able to separate the source located beside the local measurement. In both cases, the coherence algorithm shows how the LDV signal is now only correlated to the source created by the set of rods downstream.

At this location, the contribution that the disturbance created by the rods has on the autospectrum of the velocity components (u) \& (w), (figures $18(\mathrm{a}) \& 18(\mathrm{~b})$ ) can be seen in the broad peaks around $3800 \mathrm{~Hz}$. As the location of this set of rods is further away from the nozzle exit, the longitudinal velocity component (u) recorded downstream could have been expected to decrease with respect to $(\mathrm{u})$ upstream. However this is not the case. As the jet's velocity profile was unmeasured, it is unknown as to whether the measurement location downstream is within the jet's main potential core, shear layer or otherwise. In any case, as seen in 
both upstream and downstream locations, the relative position of the measurement volume respect to the rods horizontal plane, seems to have more influence than its relative location within the jet's profile.

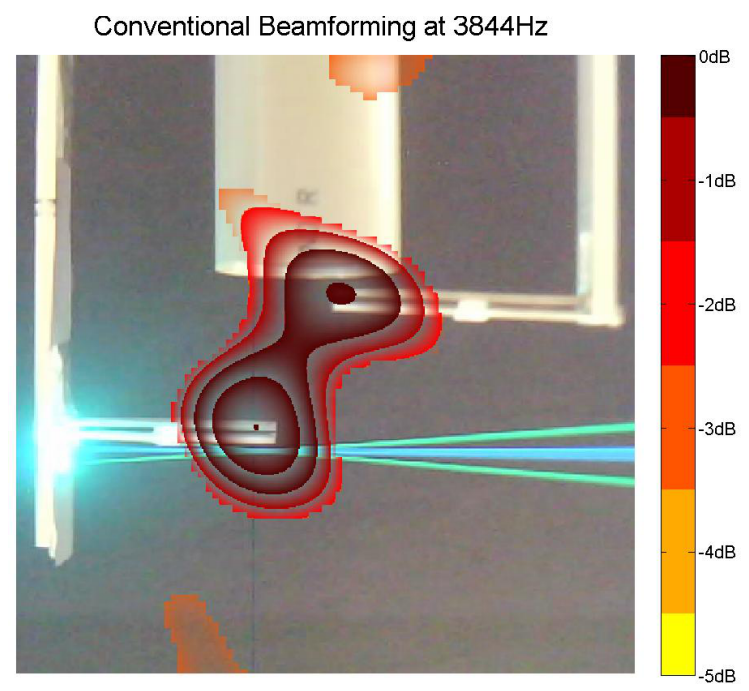

Figure 17. Conventional Beamforming

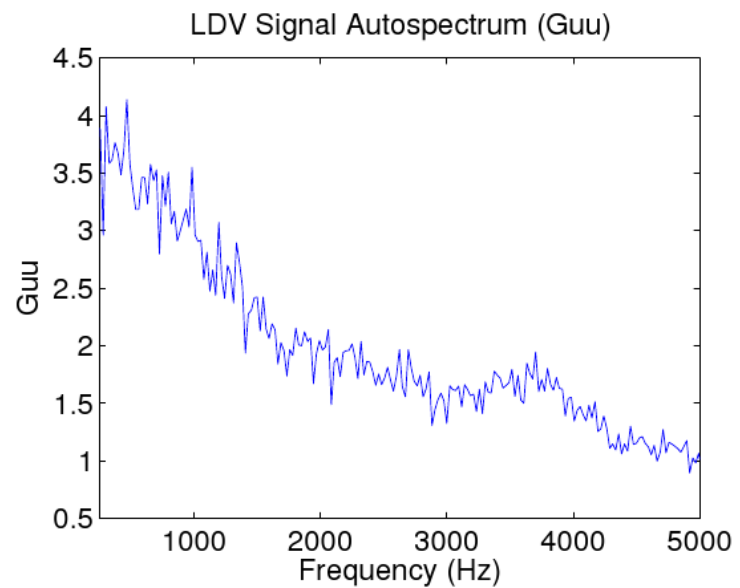

(a) Velocity autospectrum Guu

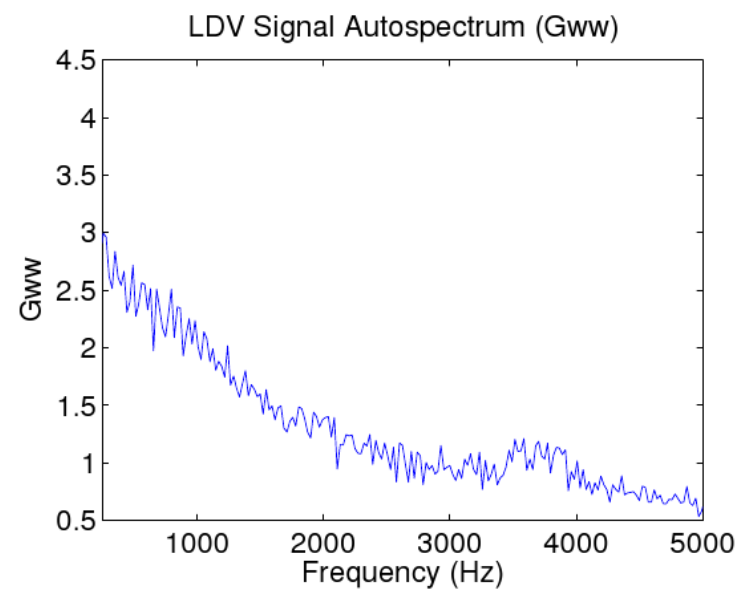

(b) Velocity autospectrum Gww

Figure 18. 


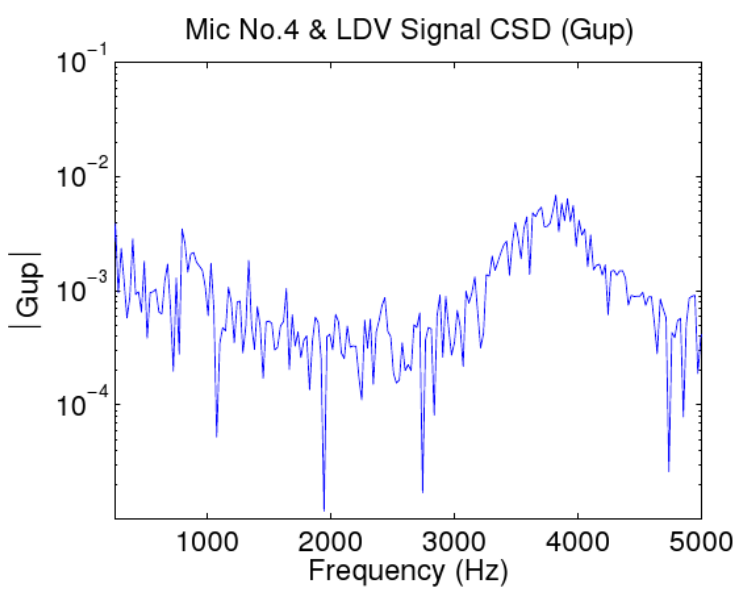

(a) Cross spectrum between the LDV (u) signal and Mic No.4

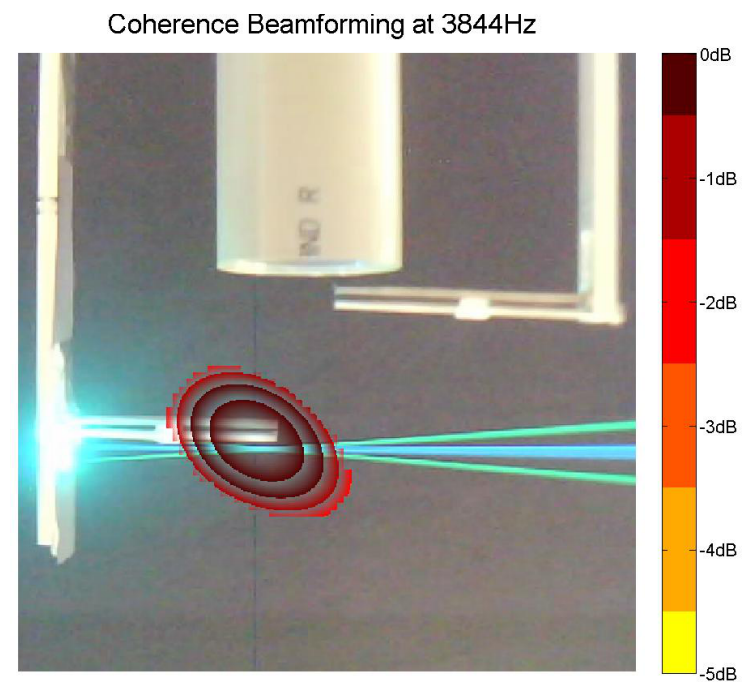

(b) Coherence Beamforming LDV (u) component used as reference signal

Figure 19.

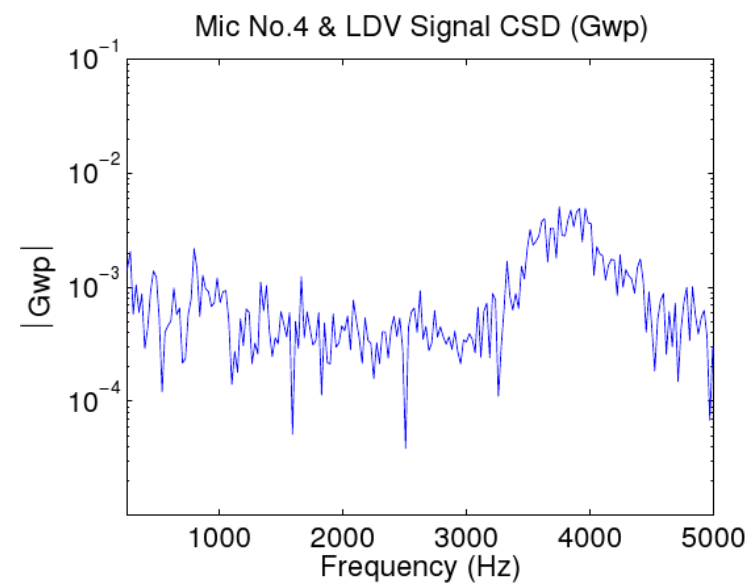

(a) Cross spectrum between the LDV (w) signal and Mic No.4

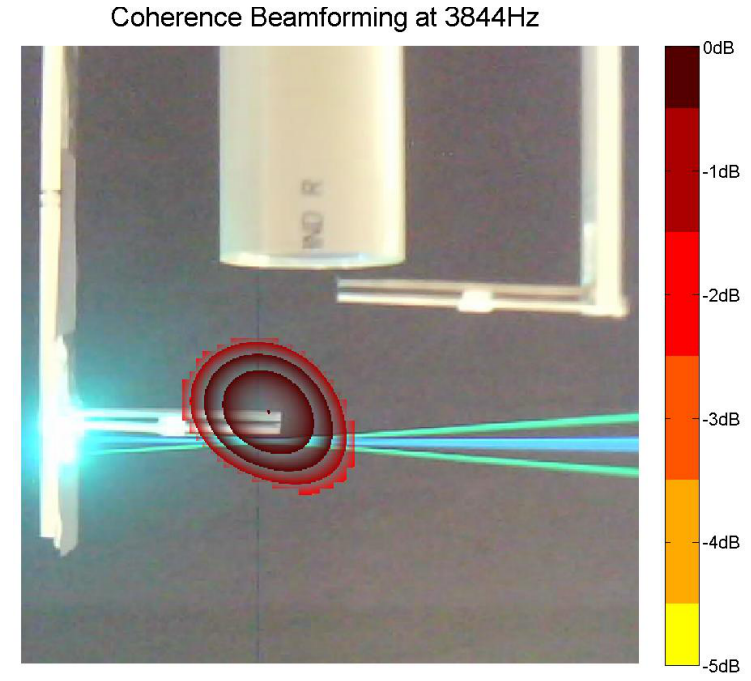

(b) Coherence Beamforming LDV (w) component used as reference signal

Figure 20.

\section{Conclusion}

A coherence based beamforming algorithm was used for the identification of aeroacoustic sources within a jet. Even in the presence of other sources, the results showed how, within a complex sound field, independent sources can be singled out when a local measurement is attainable. As this technique requires a local measurement of the area of interest, a non intrusive technique (LDV) was used to obtain measurements within the flow. Due to the nature of the LDV data acquisition, the signal processing technique necessary to combine an irregularly sampled data set and a coherence based acoustic beamforming technique was required.

\section{References}

${ }^{1}$ Chu, W., Laufer, J., and Kao, K., "Noise source distribution in subsonic jets," Inter-noise 72, 1972, pp. $472-476$. 
${ }^{2}$ Grosche, F., "Distributions of sound source intensities in subsonic and supersonic jets," Tech. rep., 1974.

${ }^{3}$ Billingsley, J. and Kinns, R., "The acoustic telescope," Journal of Sound and Vibration, Vol. 48, No. 4, 1976, pp. 485-510.

${ }^{4}$ P.Sijtsma, "Experimental techniques for identification and characterisation of noise sources (NLR)," 2004.

${ }^{5}$ Lee, H. and Ribner, H., "Direct correlation of noise and flow of a jet," The Journal of the Acoustical Society of America, Vol. 52, 1972, pp. 1280 .

${ }^{6}$ Siddon, T. E., "New correlation method for study of flow noise." 1971.

${ }^{7}$ Hurdle, P. M., Meecham, W. C., and Hodder, B. K., "Investigation of the aerodynamic noise generating region of a jet engine by means of the simple source fluid dilatation model," The Journal of the Acoustical Society of America, Vol. 56, No. 6, 1974, pp. 1708-1721.

${ }^{8}$ Siddon, T. E., "On Noise Mechanisms. Noise sources diagnostics using causality correlations," 1973.

${ }^{9}$ Schaffar, M., "Direct measurements of the correlation between axial in-jet velocity fluctuations and far field noise near the axis of a cold jet," Journal of Sound and Vibration, Vol. 64, No. 1, 1979, pp. 73-83.

${ }^{10}$ Richarz, W. G., "Direct correlation of noise and flow of a jet using Laser Doppler," AIAA Paper, Vol. 79-0571, 1979.

${ }^{11}$ Panda, J. and Seasholtz, R. G., "Experimental investigation of density fluctuations in high-speed jets and correlation with generated noise," Journal of Fluid Mechanics, Vol. 450, No. -1, 2002, pp. 97-130.

${ }^{12}$ Henning, A., Kaepernick, K., Ehrenfried, K., Koop, L., and Dillmann, A., "Investigation of aeroacoustic noise generation by simultaneous particle image velocimetry and microphone measurements," Experiments in Fluids, Vol. 45, No. 6, 2008, pp. 1073-1085.

${ }^{13}$ Siller, H., Arnold, F., and Michel, U., "Investigation of aero-engine core-noise using a phased microphone array," AIAA, 2001.

${ }^{14}$ Henning, A., Koop, L., and Ehrenfried, K., "Causality correlation in aeroacoustic experiments by means of simultaneous PIV and microphone-array url = http://bebec.eu/Downloads/BeBeC2010/Papers/BeBeC-2010-01.pdf, isbn = 978-3-00-030027$1, "$.

${ }^{15}$ Adrian, R. and Yao, C., "Power spectra of fluid velocities measured by laser Doppler velocimetry," Experiments in Fluids, Vol. 5, No. 1, 1986, pp. 17-28.

${ }^{16}$ Boyer, L. and Searby, G., "Random sampling: distortion and reconstruction of velocity spectra from fast Fourier-transform analysis of the analog signal of a laser Doppler processor," Journal of Applied Physics, Vol. 60, No. Copyright 1987, IEE, 1986, pp. 2699-707, 2837051 signal dependence velocity spectra fast Fourier-transform analysis analog signal laser Doppler processor random sampling mean sampling frequency sampling probabilities.

${ }^{17}$ Simon, L. and Fitzpatrick, J., "An improved sample-and-hold reconstruction procedure for estimation of power spectra from LDA data," Experiments in Fluids, Vol. 37, No. 2, 2004, pp. 272-280.

${ }^{18}$ Fitzpatrick, J. and Simon, L., "Estimation of cross-power spectra using sample-and-hold reconstruction of laser doppler anemometry data," Experiments in Fluids, Vol. 39, No. 6, 2005, pp. 954-965.

${ }^{19}$ Dudgeon, D., "Fundamentals of digital array processing," Proceedings of the IEEE, Vol. 65, No. 6, 1977, pp. 898-904.

${ }^{20}$ Sandro, G., "Advanced beamforming techniques in vehicle acoustics," 2010.

${ }^{21}$ Bendat, J. and Piersol, A., Random data: analysis and measurement procedures, John Wiley Sons, Inc. New York, NY, USA, 1990

${ }^{22}$ Kerherv, F., Fitzpatrick, J., and Kennedy, J., "Determination of two-dimensional space-time correlations in jet flows using simultaneous PIV and LDV measurements," Experimental Thermal and Fluid Science, Vol. In Press, Corrected Proof, 2010 . 